\title{
ANCORAGEM DE MOLARES INFERIORES COM MINI-IMPLANTES E OUTROS SISTEMAS DE ANCORAGEM: REVISÃO DA LITERATURA.
}

\section{ANCHOR MOLARS WITH MINI-IMPLANTS AND OTHER ANCHORING SYSTEMS: A LITERATURE REVIEW}

\author{
Brena Vaz Coelho* \\ Denis Clay Lopes dos Santos"**
}

\begin{abstract}
RESUMO
Este trabalho tem como objetivo realizar uma revisão de literatura sobre alguns métodos de ancoragem ortodôntica utilizadas para ancorar molares inferiores. Neste estudo, são relatadas várias pesquisas sobre técnicas e quais tipos de ancoragem utilizam, como o arco-base de Ricketts, Straight Wire com seu aparelho pré-ajustado e Edwise, entre outros. E temos como destaque o método mais atual para ancoragem, chamada de ancoragem ósseo-integrada, na qual são utilizados dispositivos como mini-implantes ou miniparafusos para sua realização. Nesta revisão, são citadas algumas vantagens e desvantagens desse novo dispositivo, como utilizá-lo, quais cuidados devem ser tomados e todos seus benefícios.
\end{abstract}

Descritores: Procedimentos de ancoragem ortodôntica

\section{ABSTRACT}

This work aims to make a literature review of some methods used for orthodontic anchorage anchor molars. In this study are reported various research techniques and what types of use as the anchor Arch Base Ricketts, Straight Wire with its device preset, and Edwise, among others. And as we highlight the most current method for anchoring, docking bone called integrated devices which are used as mini-implants or mini screws for its realization. In the cited review there are some advantages and disadvantages of this new device, how to use it, what precautions should be taken and all its benefits.

Descriptors: Orthodontic anchorage precedures

\footnotetext{
* Trabalho de Conclusão de Curso de Especialização em ortodontia e ortopedia facial. Universidade Cruzeiro do Sul - Outubro/2012

** Cirurgiã Dentista. Especialista em ortodontia e ortopedia facial. Email: brenavaz@hotmail.com

*** Professor Doutor, Mestre e PHD da Disciplina de Ortodontia e Ortopedia Facial do Programa de Graduação e Pós-Graduação do Curso de Odontologia da Universidade Cruzeiro do Sul - São Paulo. e-mail: denis.clay@cruzeirodosul.edu.br
} 
COELHO BV

SANTOS DCL

ANCORAGEM

DE MOLARES

INFERIORES

COM MINI-

IMPLANTES E

OUTROS SISTEMAS

DE ANCORAGEM:

REVISÃO DA

LITERATURA

54

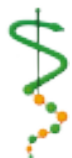

REV, ODONTOL.

UNIV. CID. SÃO

PAULO

2014; 26(1): $53-60, J A N-A B R$

\section{N T RO DUÇ ̃̃O}

A ancoragem ortodôntica tem sido uma grande preocupação para os ortodontistas desde o princípio da especialidade, pois um tratamento ortodôntico bem sucedido depende de um planejamento criterioso da ancoragem, podendo-se, assim, afirmar que este é um fator determinante para o sucesso ou insucesso do tratamento ${ }^{1}$.

Em uma terapia ortodôntica, os dentes estão expostos a forças e momentos, e essas forças atuantes geram sempre forças recíprocas da mesma magnitude, porém na direção oposta. Para evitar esses movimentos indesejados dos dentes e manter o sucesso do tratamento, tais forças recíprocas têm que ser anuladas. Ancoragem ortodôntica é a capacidade de resistir a esses movimentos indesejados, e isso pode ser conseguido através de alguns dispositivos ${ }^{2}$.

Ancoragem define-se como recurso para se evitar o movimento de um grupo de dentes enquanto são tracionados outros elementos dentários ${ }^{3}$. Já Higley ${ }^{4}$, em 1960, define essa manobra como a resistência que as estruturas dento-alveolares oferecem na alteração da forma ou posição de uma força aplicada.

Vários métodos foram utilizados pelos pesquisadores ao decorrer dos séculos, com a finalidade de conseguir ancorar os dentes posteriores, evitando-se, assim, as perdas de espaços ou mesializações indesejadas durante o tratamento ortodôntico. Essa ancoragem no setor posterior é a estabilização dos molares e posicionamento desses dentes contra qualquer movimento em todos os estágios do tratamento ortodôntico ${ }^{5}$. Conseguir um sistema de ancoragem eficaz se tornou um dos fatores mais importantes na ortodontia.

Muitas vezes, a perda dessa ancoragem pode trazer efeitos colaterais que causam o insucesso do caso. Conseguir essa estabilização dos segmentos posteriores é uma preocupação permanente em toda a história da ortodontia ${ }^{6}$.

Atualmente, o sistema de ancoragem esquelética tem sido utilizado e difundido na ortodontia por possibilitar resultados satisfatórios no controle da ancoragem, com menos incômodo do paciente, além de ser uma possibilidade para pacientes que possuem número insuficiente de dentes para outros recursos ${ }^{7}$.

Analisando a importância desse fator, este trabalho tem como objetivo, através de uma revisão da literatura, comparar diferentes sistemas de ancoragens, suas vantagens e desvantagens, focando a ancoragem em molares inferiores e a utilização desse novo recurso de ancoragem esquelética que é o mini-implante.

\section{REVISÃO DE LITERATURA}

$\mathrm{Na}$ literatura, existem algumas definições para o termo ancoragem ortodônticas. Segundo Higley ${ }^{4}$ (1960), refere-se à resistência que as estruturas dento-esqueléticas oferecem à movimentação ortodôntica. A capacidade de um dente ou grupo de dentes de resistirem a movimentos indesejáveis durante a mecânica é considerada ancoragem dentária intrabucal, segundo Ferreira $^{8}$ (1999). Também pode ser definida como resistência ao deslocamento dos dentes e é um dos fatores de suma importância para o controle da movimentação dentária9.

Em 1907, Angle ${ }^{10}$ classificou ancoragem dental em três métodos: simples, quando a resistência é obtida apoiando a força em dente ou dentes de maior tamanho e melhor localização; recíproca, quando é permitida a movimentação dental em ambos os pontos de aplicação da força; e estacionária, quando o sistema de ancoragem é rígido nos dentes de resistência, anulando movimentos de inclinação.

No decorrer dos séculos, muitos métodos foram utilizados pelos pesquisadores, com o objetivo de ancorar os dentes posteriores, para evitar as mesializações indesejadas e perdas de espaço durante o tratamento ${ }^{5}$.

Um novo conceito para ancoragem surgiu na ortodontia, denominado ancoragem esquelética, a qual não permite a movimentação da unidade de reação e é obtida devido à incapacidade de movimentação da unidade de ancoragem perante a mecânica ortodôntica. Esse tipo de ancoragem proporciona um adequado controle de forças ${ }^{11}$. 
Inicialmente, essa ancoragem esquelética era feita com implantes convencionais, porém estes apresentavam muitas limitações como a região para sua inserção, dificuldade de direcionamento para aplicação da força, gravidade da cirurgia, desconforto, tempo de espera para o início da aplicação da força, dificuldade de remoção, entre outros. Com o objetivo de superar tais limitações, foi desenvolvido um implante de tamanho reduzido que poderia ser utilizado na ancoragem ortodôntica. Este dispositivo foi denominado de mini-implante ${ }^{11}$.

Com os avanços da Odontologia, se tornou um destaque o conceito de ancoragem ósseointegrada. O uso de implantes como ancoragem temporária vem facilitando os movimentos ortodônticos ${ }^{12}$. Signori $^{13}$ em 2010 relata que se tem utilizado os miniparafusos porque apresentam resistência suficiente para suportar as forças ortodônticas, assim garantindo a ancoragem do tratamento. Em seu trabalho, fez um relato de dois casos clínicos utilizando esse dispositivo, um com a intrusão dos molares superiores para fechar mordida cruzada anterior e o outro mostrando fechamento de espaço com a mesialização do segundo e terceiro molares inferiores e, em ambos os casos, os miniparafusos se mostraram muito eficientes.

Valarelli ${ }^{14}$ em 2010 também destaca a eficácia da ancoragem esquelética nesses casos onde existe a necessidade de intrusão dos molares em pacientes adultos mutilados, pois esse procedimento com mini -implantes representa o menos invasivo e sem efeitos colaterais, como extrusão dos dentes de ancoragem. Jason ${ }^{15}$ em 2008 já destacava a possibilidade do fechamento de espaços existentes nesses pacientes, como exemplo a mesialização dos molares, quando, para tal procedimento, vários fatores têm que ser avaliados, principalmente a perda da ancoragem dos segmentos anteriores. Mas com o advento dos mini-implantes as possibilidades de sucesso nessa terapêutica aumentaram, pois esse efeito da perda de ancoragem pode ser eliminado.

Di Matteo ${ }^{12}$, em 2005, considerou que o aparelho ortodôntico consiste em dois elementos, um elemento ativo que são as partes referentes ao movimento dentário, e os elementos de resistência, os quais proporcionam a ancoragem e possibilitam esses movimentos. Esse recurso de ancoragem pode ser não dentário como, por exemplo, os mini-implantes. Porém, o autor também relata algumas desvantagens em relação ao dispositivo, como: inflamação ao redor dos miniparafusos relacionada à higiene do paciente, já que as roscas que ficam em contato com a mucosa retêm muita placa bacteriana. Como vantagem, o autor relata que forças ortodônticas podem ser aplicadas imediatamente após sua implantação.

Maia $^{16}$, em 2011, concluiu em seu trabalho, que, embora os mini-implantes sejam efetivos, possuem desvantagens, como procedimento cirúrgico, dificuldade de manutenção na higiene bucal ao redor do dispositivo, seu alto custo, risco de infecção e desconforto para o paciente.

Rothier $^{17}$, em 2009, concluiu em seus estudos que, para maior sucesso dos mini -implantes, o ideal são pacientes que não apresentem padrão de crescimento vertical, que haja um torque final de inserção ideal, distância entre as porções radiculares, inserção em gengiva inserida e em regiões mais espessas de corticais ósseas. Algumas complicações podem decorrer do uso do mini-implante, como a inflamação peri-implantar decorrente da má higienização.

Lima $^{18}$, em 2010, considerou vantajoso o tamanho reduzido do mini-implante, o que possibilita sua instalação em pontos estratégicos, resultando em uma redução dos efeitos colaterais nos dentes que, antes, serviriam para ancoragem, possibilitando, assim, prever a movimentação, reduzir o tempo de tratamento e executar movimentos difíceis, como intrusão.

Para Vilela ${ }^{19}$, em 2004, o uso desse dispositivo para ancoragem direta diminui os efeitos indesejados das forças, devido a se poder escolher o local mais indicado para sua instalação, simplificando a aparatologia ortodôntica, o que faz com que se torne uma grande opção, principalmente quando recursos extrabucais não são bem aceitos pelo paciente e também quando existe ausência de elementos dentários.

O seu mecanismo se baseia no imbri-
COELHO BV

SANTOS DCL

ANCORAGEM

DE MOLARES

INFERIORES COM

MINI-IMPLANTES

E OUTROS

SISTEMAS DE

ANCORAGEM:

REVISÃO DA

LITERATURA
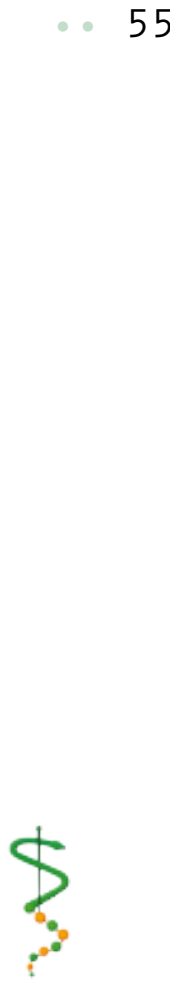

ReV. OdOntOl.

Univ. Cid. São

Paulo

2014; 26(1): 53 -

60 , JAN-ABR 
COELHO BV

SANTOS DCL

ANCORAGEM

DE MOLARES

INFERIORES

COM MINI-

IMPLANTES E

OUTROS SISTEMAS

DE ANCORAGEM:

REVISÃO DA

LITERATURA

56

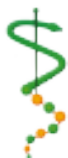

REV, ODONTOL.

UNIV. CID . SÃO

PAULO

$2014 ; 26(1):$

$53-60, J A N-A B R$
I SSN 1983-5183

camento da sua estrutura metálica no osso denso e nas corticais e não necessariamente em ossointegração. E quando ocorre, essa ossointegração dificulta a remoção, podendo aumentar os riscos de fratura. Sua instalação pode ser simples para profissionais capacitados, mas pode oferecer riscos quando mal planejada e executada. Pode-se considerar como complicações desses dispositivos o contato com as raízes dentárias vizinhas, com ou sem perfuração, a mucosite, a contaminação ou fratura. A higienização é fundamental para sua manutenção ${ }^{20}$.

Vários outros métodos de controle de ancoragem foram desenvolvidos no último século. E todos esses diferentes métodos também se mostraram eficazes para a mecanoterapia ortodôntica 5 .

Em 1941, Tweed usava arcos retangulares com algumas dobras de segunda ordem, que eram degraus no segmento posterior do arco que, quando encaixados no braquete, inclinam o longo eixo do dente para distal. Para esse sistema se tornar mais efetivo foi associado com elásticos de Classe III, ancorando então esses dentes para suportarem forças de elásticos de Classe II, sem sofrerem inclinações ${ }^{21}$.

Ricketts ${ }^{22}$, em 1983, introduziu a técnica Bioprogressiva, utilizando o osso cortical para ancoragem, por ser mais compacto, menos vascularizado e não facilmente reabsorvível. Preconizava para esse procedimento o arco base inferior, o qual incorporava torque vestibular na região dos molares enquanto produzia a expansão na região das coroas dos dentes. Para tornar essa ancoragem mais eficaz, foi incorporada no arco-base uma ativação no ângulo caudal, que produzia uma inclinação distal dos molares inferiores, para, dessa forma, resistir às forças dos elásticos intermaxilares e às forças de mesialização provenientes das alças de retração dos dentes anteriores. O arco-base ou arco-utilidade se apoiava nos incisivos, resultando nas intrusões desses dentes, sem que ocorresse a vestibularização de suas coroas.

A primeira fase do tratamento ortodôntico com extrações segundo Ricketts ${ }^{22}$ (1983) é a ancoragem dos molares, a qual pode ser máxima, onde não se permite o movimento dos molares, ou mínima, na qual o molar pode se deslocar.

O arco-utilidade básico pode ser empregado para ancorar molares inferiores, tanto nos casos de extrações quanto para evitar efeitos colaterais dos elásticos de mecânicas de Classe II, segundo Figueiredo $^{23}$ em 2008. Esse tipo de ancoragem realizada com esse dispositivo é chamado de intrabucal e cortical pois, através de um torque lingual nas coroas dos molares inferiores, desloca suas raízes para vestibular, assim tocando o osso cortical, o qual é denso, compacto, pobre em vascularização, favorece trocas metabólicas e irrigação sanguínea e, assim, neutraliza o movimento dentário ${ }^{8,23}$. Dentre outros fatores, Roth ${ }^{24}$, em 1976, afirmou que o nivelamento da curva de Spee com o arco -base favorecia a ancoragem.

Em 2005, Mustafa e Urias ${ }^{25}$ realizaram um estudo onde compararam a eficiência dos sistemas de ancoragem das técnicas Bioprogressiva e de Stright Wire testando os sistemas em dois grupos tratados com extração. Um grupo de 20 pacientes foi tratado com arco utilitário fazendo ancoragem cortical inferior e mecânica de retração para fechamentos de espaço, e o outro grupo, também com 20 pacientes, foi tratado com fio simples e com o sistema de aparelhos pré-ajustados. A avaliação do tratamento não revelou diferença significativa entre elas, no entanto a perda de ancoragem na Bioprogressiva foi de $3,1 \mathrm{~mm}$, usando apenas os molares inferiores e incisivos, e na Straight Wire houve perda de $4 \mathrm{~mm}$, mesmo com o aparelho montado em toda a arcada.

No mesmo ano, Vasconcelos ${ }^{26}$ fez uma avaliação em seu trabalho com relação à perda de ancoragem inferior também com a técnica de Stright Wire. Foram avaliadas 150 telerradiografias em norma lateral em três etapas: inicial, final de nivelamento e final de tratamento de 50 pacientes com média de idade de 13 anos e 6 meses, todos Classe I de Angle e tratados com extração dos primeiros pré-molares superiores e inferiores. Como acessório de ancoragem inferior foi utilizado o arco lingual. Com relação a esse sistema de ancoragem, foi concluído que, em pacientes dólico-faciais, os primeiros molares infe- 
riores apresentaram-se com significativa inclinação da raiz para mesial e coroa para distal, isso com relação ao seu posicionamento axial.

Outro trabalho publicado em 2005 por Vigorito ${ }^{27}$ avaliou a posição dos primeiros molares inferiores e incisivos durante a fase de nivelamento em adolescentes com má oclusão CL II $\backslash 1$ e CL I, tratados com ou sem extrações dos primeiros pré-molares, com o arco lingual como acessório de ancoragem. Os pacientes foram divididos em três grupos: Grupo I tratado com extração e uma sequência de arcos termoativados, Grupo II tratado com extração e uma sequência de arcos de aço inoxidável e Grupo III tratado sem extrações e também com uma sequência de arcos de aço inoxidável. Concluiu-se no final da avaliação que o acessório de ancoragem arco lingual fixo teve um resultado insatisfatório diante das solicitações de ancoragem na fase de nivelamento do Grupo I.

Em 2002, Vigorito e Moresca ${ }^{28}$ já haviam publicado um artigo semelhante, no qual foram utilizados os arcos linguais inferiores fixos como acessórios de ancoragem. Foram avaliados 17 pacientes com má oclusão de Classe II, divisão 1, e o tratamento foi realizado com extrações dos primeiros pré-molares superiores e inferiores. Foram utilizadas telerradiografias de norma lateral ao início e término da fase de nivelamento para realizar as comparações. E já haviam concluído, pelos resultados, que o arco lingual se apresentava insatisfatório.

Em estudos realizados em 1998, Mazari $^{29}$, comparando a perda de ancoragem entre as técnicas de arco de canto simplificado e arco reto, chegou a resultados apontando que, na primeira técnica, houve maior migração mesial do primeiro molar inferior estatisticamente significante.

Cipriano $^{6}$ em 1991 avaliou cefalometricamente o sistema de ancoragem inferior utilizando a técnica de Edgewise com angulação de braquetes. O estudo se deu através da avaliação de 200 telerradiografias obtidas de 50 pacientes portadores de má oclusão de Classe II, divisão 1, de Angle, de ambos o sexos, nas faixas etárias de 11 anos e 4 meses e 18 anos e 5 meses tratados na técnica de Edgewise com angulação dos braquetes e extração de 4 primeiros pré-molares. Através desse estudo, os autores concluíram que entre o início e o término do tratamento houve perda de ancoragem significativa do primeiro molar inferior ao nível de coroa e raiz, com inclinação áxio-mesial apical.

\section{I SCUSSÃO}

Segundo Braga 5 , muitos métodos de controle de ancoragem foram desenvolvidos no último século, auxiliando com eficiência a mecânica ortodôntica.

Outros estudos foram realizados trazendo comparações sobre a eficácia da técnica dos aparelhos pré-ajustados. Mustafá e Urias ${ }^{25}$, em 2005, compararam em suas pesquisas as técnicas de Straight Wire com a técnica Bioprogressiva de Ricketts. Concluíram que a Bioprogressiva, com a utilização do arco utilidade, usando a ancoragem cortical do setor posterior inferior, mostrou ser mais eficiente em relação à Straight Wire. Esse estudo condiz com os de Vasconcelos, que também constatou perda de ancoragem inferior com os aparelhos pré-ajustados, ainda com a utilização do arco lingual como acessório de ancoragem.

Vigorito $^{27}$ em 2005 também teve resultados insatisfatórios com o arco lingual fixo em seu trabalho com um grupo de pacientes na fase de nivelamento tratados com arcos termoativados. O que confirma os resultados em 2002 de Vigorito e Moresca, que realizaram um estudo semeIhante e já haviam concluído que o arco lingual se apresentava insatisfatório.

Como outros autores, Cipriano ${ }^{6}$, em 1991, já observava cefalometricamente em seu trabalho que a utilização do sistema de ancoragem inferior utilizando a técnica de Edwise com angulação de braquetes se mostrava ineficiente com perda de ancoragem significativa entre o início e término dos tratamentos.

Mazari ${ }^{29}$ em 1998 comprovou perda de ancoragem do arco de canto simplificado com a migração mesial do primeiro molar inferior em comparação com a técnica de arco reto.

Embora Ricketts ${ }^{22}$ em 1976 tenha intro-
COELHO BV

SANTOS DCL

ANCORAGEM

DE MOLARES

INFERIORES COM

MINI-IMPLANTES

E OUTROS

SISTEMAS DE

ANCORAGEM:

REVISÃO DA

LITERATURA

\section{7}

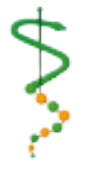

Rev. OdOntOl.

UnIV. CID. São

Paulo

2014; 26(1): 53 -

60, JAN-ABR 
COELHO BV

SANTOS DCL

ANCORAGEM

DE MOLARES

INFERIORES

COM MINI-

IMPLANTES E

OUTROS SISTEMAS

DE ANCORAGEM:

REVISÃO DA

LITERATURA

REV, ODONTOL

UNIV. CID. SÃO

$2014 ; 26(1)$ :

$53-60, J A N-A B R$ duzido o arco utilidade tendo como base a ancoragem cortical e esse método tenha apresentado muito efetivo de acordo com as pesquisas, um novo conceito de ancoragem ósseo-integrada vem ganhando espaço dentro do cenário ortodôntico.

Antes, esse novo conceito era realizado com implantes convencionais, mas estes se mostraram muito limitados por apresentarem dificuldades para inserção, gravidade cirúrgica e tempo de espera para realizar a força, entre outros. Então foi desenvolvido um dispositivo com menores dimensões, denominado mini-implante, como relatou Araújo ${ }^{11}$ em 2008.

Esse novo método que utiliza os miniparafusos apresenta resistência suficiente para suportar as forças ortodônticas e tais forças podem ser aplicadas imediatamente após sua instalação, segundo Signori ${ }^{13}$, 2010 e Di Matteo ${ }^{12}$, 2005. Trata-se de uma ancoragem temporária que facilita os movimentos ortodônticos. Além do mais, Lima $^{18}$ em 2010 e Vilela ${ }^{19}$, em 2004, consideraram vantajoso o reduzido tamanho do mini-implante, que pode ser instalado em pontos estratégicos, assim reduzindo os efeitos colaterais dos dentes e o tempo de tratamento executando movimentos difíceis. Vilela ainda diz ser uma grande opção por ter boa aceitação pelos pacientes e ser eficiente em casos de ausência dentária. Janson ${ }^{15}$ em 2008 confirma que essa técnica pode realizar movimentos difíceis, como mesialização dos molares sem causar efeitos indesejáveis, concordando com Vilela ${ }^{19}$. Outro tipo de movimentação complicada que pode ser facilmente realizada com a utilização dos mini-implantes é a intrusão dos molares, segundo Valarelli ${ }^{14}$.

Embora sejam grandes as vantagens desse novo sistema, também são coincidentes as opiniões dos autores em relação às desvantagens dos miniparafusos. Di Matteo ${ }^{12}$, Freire-Maia ${ }^{16}$, Rothier ${ }^{17}$ e
Consolaro ${ }^{20}$ são unânimes quando se trata da higienização do mini-implante. Se mal realizada, pode causar inflamação ao redor do dispositivo, as inflamações peri -implantares. Outras desvantagens segundo esses autores é que, se a cirurgia for mal planejada, o dispositivo pode entrar em contato com as raízes dentárias vizinhas, com ou sem perfuração, e também pode causar mucosites, contaminação e fraturas. Além de ser um procedimento cirúrgico, possuir alto custo, risco de infecção e desconforto para o paciente.

\section{CONCLUSÃO}

Ancoragem é um dos fatores de maior importância em ortodontia. Muitos são os métodos para realizar esse procedimento em molares inferiores. Umas das grandes opções na atualidade são os dispositivos denominados mini-implantes, com um novo conceito de ancoragem ósseo-integrada. Estes possuem muitos benefícios, como facilitarem os movimentos ortodônticos, diminuírem o tempo do tratamento, possibilitarem a realização de forças imediatamente após sua instalação e não dependerem do paciente, além de serem bem aceitos por este. Outra grande vantagem é a de se poder utilizá-los em regiões com ausências dentárias. Também temos que levar em consideração, antes de optar por esse método, as questões de higiene, para evitar inflamações em volta do dispositivo, o desconforto para o paciente, além de se tratar de um procedimento cirúrgico e ter um alto custo.

Embora o método dos mini-implantes seja um avanço na ortodontia, não podemos esquecer que ainda existem muitos outros artifícios de ancoragem que foram desenvolvidos em toda a história, dando para os profissionais uma gama de possibilidades de escolha, de acordo com suas indicações para o tratamento que será realizado. 
1. Araújo TM, Nascimento MHA, Bezerra F, Sobral MC. Ancoragem esquelética em Ortodontia com mini-implantes. Revista Dental Press de Ortodontia e Ortopedia Facial 2006 11(4):126-56.

2. Feldmann I, Bondemark L. Orthodontic anchorage: a systematic review. Angle Orthod 2006 May;76(3):493501.

3. Nanda R. Biomechanics in clinical orthodontics. Philadelphia: Saunders; 1997.

4. Higley LB. Anchorage in orthodontics. Am J Orthod 1969 Jun;55(6):791-4.

5. Braga C, Hoffelder L, Marchioro E, Berthold T. Ancoragem Ortodôntica Rev Odonto Ciência 2002 17(38):3808.

6. Cipriano R, Vigorito JW. Estudo cefalométrico-radiográfico do sistema de ancoragem inferior empregado na técnica do arco de canto com angulação de braquetes nos segmentos posteriores. Ortodontia 1991 maio-ago;24(2):39-52.

7. Squeff LR, Simonson MBA, Elias $\mathrm{CN}$, Nojima LI. Caracterização de mini -implantes utilizados na ancoragem ortodôntica. Revista Dental Press de Ortodontia e Ortopedia Facial 2008 set-out;13(5):49-56.

8. Ferreira F. Ortodontia: diagnóstico e planejamento clínico. 3 ed. São Paulo: Artes Médicas; 1999.

9. Graber T. Ortodontia: princípios e técnicas atuais. 2 ed. Rio de Janeiro: Artes Médicas; 1996.

10. Angle E. Treatment of malocclusion of the teeth. Philadelphia: S.S. White Dental; 1907.

11. Araújo L, Zenóbio E, Vilaça F, Pacheco W, Cosso M. Evolução dos implantes na ancoragem ortodôntica. Arq Bras Odontol 2008 4(1):28-31.
12. Di Matteo RC, Villa N, Sendyk WR. Movimentação de molares inferiores ancorados em miniparafusos. Revista Dental Press de Ortodontia e Ortopedia Facial 2005 jul-ago; 10(4):124-33.

13. Signori D, Gross J, Oliveira J, Nascimento Jr R, Gross A. Ancoragem Ortodôntica com miniparafusos: relato de casos clínicos. Rev Assoc Paul Cir Dent 2010 nov-dez;64(6):415-20.

14. Valarelli FP, Reys Celi MV, Chiqueto KFG, Freitas KMS, Valarelli DP. Efetividade dos mini-implantes na intrusão de molares superiores. Innov Implant J, Biomater Esthet 2010 jan -abr;5(1):66-71.

15. Janson M, Silva DAF. Mesialização de molares com ancoragem em mini-implantes. Revista Dental Press de Ortodontia e Ortopedia Facial 2008 set-out;13(5):88-94.

16. Freire-Maia B, Pereira TJ, Ribeiro MP. Distalização de segundo molar inferior impactado através da utilização de ancoragem esquelética com miniplaca: relato de caso. Rev Dental Press J Othod 2011 jul-ago;16(4):132-6.

17. Rothier EKC, Vilella OV. Ancoragem Ortodôntica com mini-implantes: fatores de sucesso. Rev Bras Odontol 2009 ju-dez;66(2):177-82.

18. Lima LAC, Lima C, Lima V, Lima V. Mini-implante como ancoragem absoluta: ampliando os conceitos de mecânica ortodôntica. Innov Implant J, Biomater Esthet 2010 5(1):85-91.

19. Villela H, Villela P, Bezerra F, Labiossiérie Jr MA, Soares AP. Utilização de mini-implantes para ancoragem ortodôntica direta. Innovations J 2004 8(1):5-12.

20. Consolaro A, Sant'ana E, Francischone Jr CE, Consolaro MFM-O, Barbosa BA. Mini-implantes: pontos consensuais e questionamentos sobre o seu uso clínico. Revista Dental Press de Ortodontia e Ortopedia Facial 2008 set-out;13(5):20-7.
SANTOS DCL

ANCORAGEM DE MOLARES INFERIORES COM MINI-IMPLANTES E OUTROS

SISTEMAS DE

ANCORAGEM:

REVISÃO DA

LITERATURA

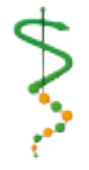

Rev. OdOntOl. Univ, Cid, São PAulo

$2014 ; 26(1): 53$. 60 , JAN-ABR 
COELHO BV

SANTOS DCL

ANCORAGEM

DE MOLARES

INFERIORES

COM MINI-

IMPLANTES E

OUTROS SISTEMAS

DE ANCORAGEM:

REVISÃO DA

LITERATURA
21. Proffit WR. Ortodontia contemporânea. 2 ed. Rio de Janeiro: Guanabara; 1995.

22. Ricketts RM. Técnica bioprogressiva de Ricketts. Buenos Aires: Médica Panamericana; 1983.

23. Figueiredo MA, Figueiredo CTP, Nobuyasu M, Gondo GY, Siqueira DF. A versatilidade clínica do arco utilidade. Revista Dental Press de Ortodontia e Ortopedia Facial 2008 jul-ago;13(4):127-56.

24. Roth RH. Five year clinical evaluation of the Andrews straight-wire appliance. J Clin Orthod 1976 Nov;10(11):83650.

25. Urias D, Mustafa FI. Anchorage control in bioprogressive vs straightwire treatment. Angle Orthod 2005 Nov;75(6):987-92.

26. Vasconcelos MHF, Ferreira DP, Trivino T, LA. M. Avaliação cefalométrica da perda de ancoragem inferior, em pacientes meso e dólicofaciais tratados com a técnica straight wire e extração de pré-molares. Rev Odonto 2005 13(25):42-51.
27. Vigorito JW, Dominguez GC, Tortamano A. Nivelamento-controle da ancoragem durante a fase de alinhamento e nivelamento em casos com extrações e sem extrações utilizada a técnica MB. Ortodontia 2005 38(2):110-18.

28. Vigorito JW, Moresca R. Estudo comparativo cefalométrico radiográfico sobre os efeitos dos arcos termoativos na estabilidade dos dentes posteriores inferiores, durante a fase de nivelamento, utilizando-se do arco lingual fixo e prescrição MTB. Ortodontia 2002 35(3):57-66.

29. Mazari R. Estudo cefalométrico da perda de ancoragem inferior, durante a fase de nivelamento, nas técnicas de arco de canto simplificado e arco reto [Dissertação]. Bauru: USP; 1998.

Recebido: 26/11/2013

Aceito: 02/02/2014 